journal club

Herzinsuffizienz

\title{
Diabetes wirkt als BNP-Booster
}

Fragestellung: Unterscheiden sich die Werte des B-Typ-natriuretischen Peptids (BNP) von herzinsuffizienten Patienten mit Diabetes von denen herzinsuffizienter Patienten ohne Diabetes?

Hintergrund: Die chronische Herzinsuffizienz geht mit einer erhöhten neurohumoralen Aktivität einher. Das Neurohomon BNP wird primär von den Herzventrikeln sezerniert. BNP-Konzentrationen korrelieren mit dem Grad der ventrikulären Dysfunktion, sodass sie als einfach zu bestimmender diagnostischer Marker für die Herzinsuffizienz genutzt werden. BNP ist bei Diabetikern nicht nur mit der diastolischen Dysfunktion sondern auch mit dem kardiovaskulären und Mortalitätsrisiko assoziiert.

In einigen Studien wurde bereits herausgefunden, dass die BNP-Werte bei herzinsuffizienten Patienten mit Diabetes höher waren als bei Patienten ohne Diabetes, aber gute kontrollierte Studien lagen dazu bisher nicht vor $[1,2]$. Deshalb sollte in der vorliegenden Studie unter Beachtung der Parameter Alter, Hypertonie, linksventrikuläre Ejektionsfraktion, Wanddicke und NYHA-Schweregrad der Einfluss des Diabetes auf die BNPKonzentrationen bei herzinsuffizienten Patienten genauer untersucht werden.

Patienten und Methodik: Von den 559 Patienten hatten 276 eine KHK, 234 eine hypertensive Herzerkrankung und 49 Patienten eine dilatative Kardiomyopathie. 175 Diabetiker nahmen

Peng Q, Hu W, Su H et al. Levels of B-type natriuretic peptide in chronic heart failure patients with and without diabetes mellitus,

EXPERIMENTAL AND THERAPEU-

TIC MEDICINE 2013;5:229-32. teil, 384 Patienten hatten keinen Diabetes mellitus.

Die arterielle Hypertonie wurde in die drei Schweregrade 140, 160, 180 bzw. 90, 100, $110 \mathrm{mmHg}$ eingeteilt. Ein Diabetes wurde nach Medikation, Nüchtern-BZ> 7,0 mmol/1 oder $2 \mathrm{~h}$ postprandialem $\mathrm{BZ}>$
$11,0 \mathrm{mmol} / \mathrm{l}$ definiert. Der BNP-Wert wurde morgens nach $12 \mathrm{~h}$ Fasten bestimmt. Aus allen Parametern wurde ein Score zwischen 4 und 14 Punkten für jeden Patienten errechnet.

Ergebnisse: Die Patienten waren im Mittel 68,3 bzw. 69,5 Jahre alt; ihr BMI lag bei 25,6 bzw. $23.3 \mathrm{~kg} / \mathrm{m}^{2}$. Der Blutdruck der Patienten lag im Mittel bei 142,3 bzw. 139,2 und 80,6 bzw. 78,0 mmHg. Die EF war 55,6 bzw. 57,2, die LVW 10,1 bzw. 10,2 mmHg. Das Verhältnis der NYHA-Stadien II: III: IV war bei Diabetikern $(\mathrm{n}=175)$ 84:71:20 und bei Patienten ohne Diabetes $(n=384)$ 189:159:36. Der BNP-Wert lag bei Diabetikern höher $(1144 \mathrm{ng} / \mathrm{l})$ als bei Nichtdiabetikern $(884 \mathrm{ng} / \mathrm{l} ; \mathrm{p}<0,05)$, was auch für alle drei NYHA-Stadien galt. Die BNP-Werte waren bei den Diabetikern mit Risiko-Score-Punkten zwischen 9 bis 13 signifikant höher $(\mathrm{p}<0,05)$ als bei Nichtdiabetikern.

Schlussfolgerung: Die BNP-Konzentrationen steigen in beiden Gruppen mit dem NYHA-Stadium, bei Diabetikern sind sie aber höher als bei Nichtdiabetikern. Zu wenige Patienten mit NYHA-IV nahmen teil, um signifikante Unterschiede zu erzielen. Es hatten aber mehr Diabetiker mit NYHA-IV höhere BNP-Werte als $4000 \mathrm{ng} / \mathrm{l}$.

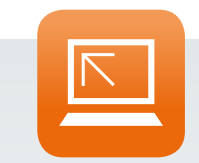

Weitere Beiträge zum Thema „Herzinsuffizienz" finden Sie online im Dossier unter. >www.springermedizin.de/ 215376

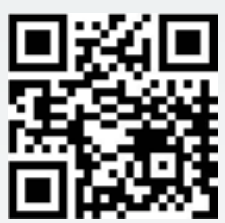

\section{-Kommentar von Prof. Dr. Emanuel Fritschka}

\section{Vielleicht liegt das an den höheren Blutzuckerwerten}

Die Studie ergab, dass bei Diabetikern weitere Faktoren die BNP-Werte beeinflussen. Möglicherweise wirken die sich auf die BNP-Produktion und Degradation aus. Da die Nierenfunktion nicht anders war, dürften die Unterschiede mehr der verstärkten Produktion von BNP zuzuschreiben sein. Diabetiker mit KHK haben höhere BNP-Werte als Patienten mit KHK und ohne Diabetes. Letztlich ist es auch möglich, dass höhere Blutzuckerwerte mit der Tendenz zu höherem Plasmavolumen die BNP-Produktion bei Diabetikern tendenziell verstärkt haben.

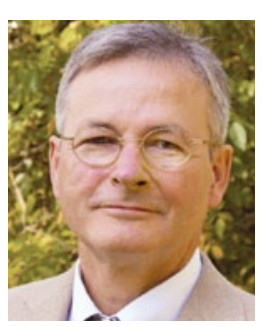

Prof. Dr. med. Emanuel Fritschka

Sinntalklinik, Rehabilitations- und AHB-Klinik für Nephrologie und Diabetologie der DRV Nordbayern Wernarzerstr. 12, 97769 Bad Brückenau Emanuel.fritschka@drv-nordbayern.de

\footnotetext{
Literatur:

1. Boonman LJ, Rutten FH, Cramer MJ et al. BMC Public Health 2009;21:479-52.

2. Igarashi M, Jimbu Y, Hiram A and Tominaga M Endocr J 2005;52: 352-62
} 